\title{
Síndrome de Miller Fisher: una variante poco común de Guillain Barré
}

\author{
Miller Fisher Syndrome: An uncommon Guillain Barré variant
}

\author{
Ana Leticia Morales-Galindo,* Luis Fernando Bolaños-Aparicio ${ }^{\ddagger}$ \\ * Servicio de Pediatría; ${ }^{\ddagger}$ Médico Residente de tercer año de Pediatría. UMAE Hospital de Pediatría, \\ Centro Médico Nacional Siglo XXI, Instituto Mexicano del Seguro Social. Ciudad de México.
}

\begin{abstract}
RESUMEN
Objetivo: Presentar el caso clínico de un paciente de 10 años con diagnóstico de síndrome de Miller Fisher, variante del síndrome de Guillain Barré, lo cual es raro en niños. Descripción del caso: Acude con oftalmoplejía, diplopía, debilidad muscular y ataxia; el diagnóstico de síndrome de Miller Fisher se realizó con base en los datos clínicos, presencia de disociación albúmino-citológica en el líquido cefalorraquídeo, y por el reporte de estudio electroneurográfico con neuroconducción motora anormal y polirradiculoneuropatía de tipo axonal. El paciente recibió gammaglobulina inmune intravenosa con lo que mostró mejoría de la sintomatología. Conclusión: El cuadro clínico debe orientar a la sospecha de síndrome de Miller Fisher, pero se deberán realizar estudios para establecer el diagnóstico y ofrecer tratamiento lo más pronto posible.
\end{abstract}

Palabras clave: Miller Fisher, Guillain Barré, oftalmoplejía, ataxia, polirradiculoneuropatía, neuroconducción.

\section{INTRODUCCIÓN}

El síndrome de Miller Fisher fue reconocido por primera vez en 1932 por James Collier como una tríada clínica de oftalmoplejía, a taxia y arreflexia. Más tarde, en 1956 Charles Miller Fisher lo reportó como una variante limitada del síndrome de Guillain Barré. ${ }^{1}$ Por lo general, este síndrome de comienza a lo largo de

\begin{abstract}
Objective: We present the clinical case of a 10-yearold patient with a diagnosis of Miller Fisher syndrome, a variant of Guillain Barré syndrome, which is rare in children. Case description: Ophthalmoplegia, diplopia, muscle weakness, and ataxia was the patient's clinical picture. Diagnosis of Miller Fisher syndrome was made based on the clinical data, the presence of albumin-cytological dissociation in the cerebrospinal fluid, and the report of an electroneurographic study with abnormal motor neuronconduction and polyradiculoneuropathy of the axonal type. The patient received intravenous immune gamma globulin showing improvement in symptoms. Conclusion: The clinical picture should guide the suspicion of Miller Fisher syndrome, but studies should be carried out to confirm the diagnosis, in order to offer treatment as soon as possible.
\end{abstract}

Keywords: Miller Fisher, Guillain Barré, ophthalmoplegia, ataxia, poly-radiculoneuropathy, neuroconduction.

los días con el rápido desarrollo de tres problemas que constituyen la tríada clínica, debilidad de los músculos dentro o alrededor del ojo, presencia de movimientos anormales, descoordinados y mal equilibrio con la marcha torpe, así como la pérdida de los reflejos tendinosos profundos.

Existe un predominio en el sexo masculino en una proporción de $2: 1$ y se ha reportado en una edad media

Correspondencia: Ana Leticia Morales-Galindo, E-mail: anaicitel@hotmail.com

Citar como: Morales-Galindo AL, Bolaños-Aparicio LF. Síndrome de Miller Fisher: una variante poco común de Guillain Barré. Rev Mex Pediatr. 2021; 88(4): 149-151. https://dx.doi.org/10.35366/102779 
de inicio de 43.6 años, aunque se han notificado casos de síndrome de Miller Fisher a cualquier edad. Los casos pediátricos descritos en la literatura son raros. ${ }^{2,3}$

El objetivo es presentar el caso de un niño de 10 años con diagnóstico clínico de síndrome de Miller Fisher.

\section{PRESENTACIÓN DEL CASO}

Paciente masculino de 10 años sin antecedentes heredofamiliares ni perinatales de importancia. Esquema de vacunación completo para la edad.

Niega infecciones respiratorias o gastrointestinales previo al inicio del padecimiento, el cual inició con edema palpebral izquierdo y, 24 horas después fue bipalpebral, agregándose ptosis, y vómito de contenido gástrico, de predominio matutino. Por los familiares se refiere cefalea frontal de intensidad moderada a intensa, alteración en la emisión del lenguaje, y diplopía. Niegan presencia de movimientos anormales.

Examen físico: poco cooperador, funciones mentales alteradas por la emisión del lenguaje, obedece órdenes simples, cráneo normocéfalo. Pares craneales I: sin alteraciones, II: identifica objetos, III, IV, VI: mirada primaria normal, pupilas isocóricas de $3 \mathrm{~mm}$, normorreflécticas, ducciones, versiones y vergencias alteradas, no logra movilidad ocular, oculocefálicos horizontales y verticales abolidos. Par V: sensibilidad de V1, V2 y V3 sin alteraciones, masetero con tono y fuerza normal y simétrica, reflejo maseterino presente. El VII: ptosis palpebral bilateral, asimetría facial al reposo del lado izquierdo, sin gesticulación. VIII: escucha; IX y X: úvula central, reflejo nauseoso y tusígeno presente, no eleva el velo del paladar; XI: fuerza en esternocleidomastoideos y trapecios simétrica. Par XII: lengua central al reposo y a la protrusión. En cuanto a los movimientos generales, se observó trofismo global normal, movimientos pasivos y activos sin alteraciones, tono en las cuatro extremidades normal (global 0/4 en escala de Ashworth), fuerza muscular 5/5 en la escala de Daniels; REMS patelar y aquileo abolido global, REMS bicipital y tricipital y estiloradial: uno de cuatro global, respuesta plantar flexora bilateral. No se valoró marcha, pues el paciente se encontraba en reposo (Figura 1).

Líquido cefalorraquídeo (LCR): aspecto transparente, células $3 / \mathrm{mm}^{3}$, leucocitos $3 / \mathrm{mm}^{3}$, eritrocitos 0 , glucosa $59.2 \mathrm{mg} / \mathrm{dL}$, proteínas $127 \mathrm{mg} / \mathrm{dL}$, deshidrogenasa láctica $32 \mathrm{U} / \mathrm{L}$, lactato $15.38 \mathrm{mg} / \mathrm{dL}$. No se observaron bacterias, eritrocitos ni levaduras.
Reporte clínico de estudio electroneurográfico: neuroconducción motora anormal; conclusión: polirradiculoneuropatía de tipo axonal.

Aspectos éticos: la presentación de este caso y contenido gráfico se realiza contando con el consentimiento informado del padre y asentimiento informado del paciente.

\section{DISCUSIÓN}

El síndrome de Miller Fisher (SMF) es una enfermedad aguda autoinmune desmielinizante con afección de los nervios craneales y periféricos, considerado como una variedad de presentación clínica del síndrome de Guillain Barré..$^{4,5}$

En el síndrome de Miller Fisher la característica más común es diplopía (78\%), seguido de ataxia (48\%); en el 34\% de los casos se presentan ambas. Los síntomas menos frecuentes consisten en disestesia de extremidades, blefaroptosis, parálisis facial, bulbar y pupilar; debilidad motora leve, y alteración en la micción. Estos signos clínicos están precedidos de signos de infección del tracto respiratorio superior entre 56 y $76 \%$ de los pacientes. Los patógenos más comunes son Campylobacter jejuni y Haemophilus influenzae; sin embargo, Mycoplasma pneumoniae y citomegalovirus también se encuentran asociados.

Aunque en el paciente no se refieren infecciones respiratorias ni digestivas previas, su inicio fue agudo, lo que coincide con lo reportado en la literatura.

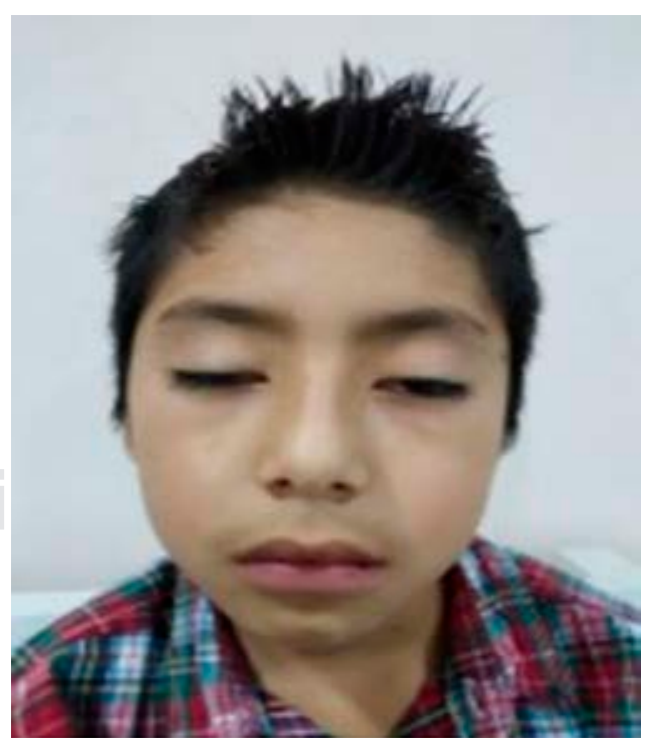

Figura 1: Oftalmoplejía en paciente con síndrome de Miller Fisher. 
Habitualmente estos pacientes inician con síntomas neurológicos, en promedio, de ocho a 10 días (variación de uno a 30 días) luego de la enfermedad precedente. La enfermedad progresa hasta que se alcanza un acmé clínico de alrededor de una semana (de dos a 21 días), después de los síntomas neurológicos iniciales.

Se puede hacer diagnóstico de síndrome de Miller Fisher con la historia clínica compatible, los hallazgos normales en las imágenes por tomografía de cráneo o resonancia magnética, así como en la presencia de disociación albumino-citológica en líquido cefalorraquídeo.

Los anticuerpos anti-GQ1b que actúan contra GQ1b (un componente gangliósido de los nervios) bloquean la liberación de acetilcolina de las terminales nerviosas motoras. Se relaciona con la actividad de la enfermedad y se puede utilizar como marcador de diagnóstico en síndrome de Miller Fisher. No es exclusivo, pero ayuda para la confirmación. ${ }^{6}$

Los resultados de los estudios electrofisiológicos muestran afectación nerviosa periférica como anomalías en las latencias motoras distales, velocidades de conducción motora o velocidades de conducción sensorial. ${ }^{7}$ En el caso presentado se realizó el diagnóstico con base en la historia clínica, las manifestaciones clínicas, los hallazgos tomográficos y la disociación albuminocitológica, y se complementó con electromiografía.

El tratamiento de síndrome de Miller Fisher es con inmunoglobulina intravenosa y plasmaféresis, como en el usado en Guillain Barré clásico. ${ }^{8}$ Nuestro paciente fue tratado con inmunoglobulina intravenosa a dosis de $1.3 \mathrm{~g} / \mathrm{kg}$, con mejoría de la disartria y diplopia.

\section{AGRADECIMIENTOS}

$\mathrm{Al}$ padre del paciente y al paciente por permitir su abordaje, exploración y toma de fotografías.

\section{REFERENCIAS}

1. Bukhari S, Taboada J. A case of miller fisher syndrome and literature review. Cureus. 2017; 9(2): 1048.

2. Jiménez-Domínguez R, Montante-Montes-De A, Martínez-Mata J, Cruz-Fino D, Ruíz-Franco A, Ramírez-Hernández $M$ et al. Síndrome de Miller Fisher, una variante del síndrome de Guillain Barré. Rev Mex Neuroci. 2009; 10(5): 367-370.

3. Sánchez-Torrent L, Noguera-Julian A, Pérez-Dueñas B, Nascimento-Osorio A, Colomer-Oferil J. Síndrome de Miller Fisher en la edad pediátrica: descripción de 3 casos. An Pediatr (Barc). 2009; 71(4): 377-378.

4. Govoni V, Granieri E, Tola MR, Casseta I, Ruppi P, Vaghi L. The frequency of clinical variants of Guillain-Barré syndrome in Ferrara, Italy. J Neurol. 1999; 246: 1010-1014.

5. Bassal F, Lupo P. Case 3: Ophthalmoplegia and Unsteady Gait in an 11-year-old Boy. Pediatr Rev. 2018; 39(1): 39.

6. Muñiz AE. Multiple cranial nerve neuropathies, ataxia and, areflexia: Miller Fisher syndrome in a child and review. Am J Emerg Med. 2017; 35(4): 661.e1-661.e4.

7. De Pablos C, Calleja J, Fernández F, Berciano J. Miller Fisher syndrome: an electrophysiologic case study. Electromyogr Clin Neurophysiol. 1988; 28(1): 21-25.

8. Mori M, Kuwabara S, Fukutake T, Hattori T. Intravenous immunoglobulin therapy for Miller Fisher syndrome. Neurology. 2007; 68(14): 1144-1146.

Conflicto de intereses: los autores declaran que no tienen.

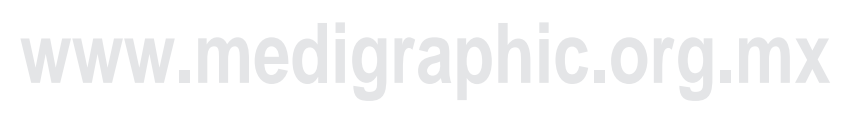

